\title{
Continuum Theory of Biaxial Nematic Liquid Crystals
}

\author{
I. W. Stewart \\ Department of Mathematics and Statistics \\ University of Strathclyde \\ Livingstone Tower \\ 26 Richmond Street \\ Glasgow G1 1XH \\ United Kingdom
}

August 8, 2014

e-mail: i.w.stewart@strath.ac.uk 


\section{Introduction}

The possibility of a biaxial nematic phase was first proposed in 1970 by Freiser $[1,2]$ while the first experimental results were reported in 1980 by $\mathrm{Yu}$ and Saupe [3]. This was followed by other early reports on the synthesis of thermotropic biaxial nematics (see, for example, [4-7]) and a review and discussion of such materials was given by Luckhurst [8].

The first attempt at a continuum theory was by Saupe [9], with other formulations following by Liu [10], Kini [11], Govers and Vertogen [12-14], Chauré [15] and Leslie, Laverty and Carlsson [16]. These authors essentially obtained the same continuum theory from differing approaches and viewpoints, as mentioned by Leslie and Carlsson [17] when they examined theoretically flow alignment in a biaxial phase. The formulation by Leslie et al. [16] is slightly more general and therefore it is this theory that will be adopted here. There has been a resurgent interest in the continuum modelling of biaxial nematic liquid crystals, largely due to emerging experimental results that have appeared in the literature, especially those motivated by more recent confirmation of biaxial nematic phases [18-22]. A series of comments on some of these results has been made by Luckhurst [23].

This review will follow the notation and terminology used by Leslie et al. [16, 24] and Carlsson et al. $[25,26]$. Section 2 introduces the basic mathematical description of the isothermal continuum model for an incompressible biaxial nematic liquid crystal; a summary of the appropriate elastic, magnetic and electric energies will also be given. Section 3 reviews the general continuum equations. After introducing some elementary concepts in Sections 3.1 and 3.2 for modelling the dynamics of the biaxial nematic phase, Section 3.3 presents the dynamic equations. An example for a model of simple shear flow is given in Section 3.5. Section 4 reviews the equilibrium equations and Section 4.2 gives an example of how they can be used to identify possible sample alignments that can be induced by an externally applied magnetic field. The chapter closes with a brief conclusion in Section 5 .

\section{Continuum model and energies}

It proves convenient to introduce the schematic diagram of a biaxial plate to describe the necessary directors for a biaxial nematic and to this end we follow the description used by Carlsson and Leslie [27]. This will also facilitate a clear description for the associated magnetic susceptibilities and dielectric permittivities. Cartesian suffix notation will be used where appropriate. A comma denotes partial differentiation with respect to the variable it precedes and the Einstein summation convention will be assumed: if a suffix appears twice in an expression, and only twice, then the expression is to be summed over the repeated suffix from one to three.

Without loss of generality, the symmetry of the biaxial phase may be visualised as a regular rectangular plate with sides of lengths $a, b$ and $c$ with $a>b>c$. In reality, the local molecular shape may well be plate-like or lozenge-like, but many other shapes of molecule are feasible such as cross-shaped, bone-shaped and boomerangshaped molecules $[8,22,23]$. In addition, the molecular shape itself may change becuase of its flexibility. Nevertheless, this does not really change the basic mathematical de- 
scription at the continuum level since it is the identification of two optic axes that is important because these signify the presence of the phase biaxiality. The orientation of this plate can be described via three mutually orthogonal unit vectors $\mathbf{n}, \mathbf{m}$ and $\mathbf{l}$ that depend on the spatial variable $\mathbf{x}$ and time $t$, as shown in Fig. 1. In this description,

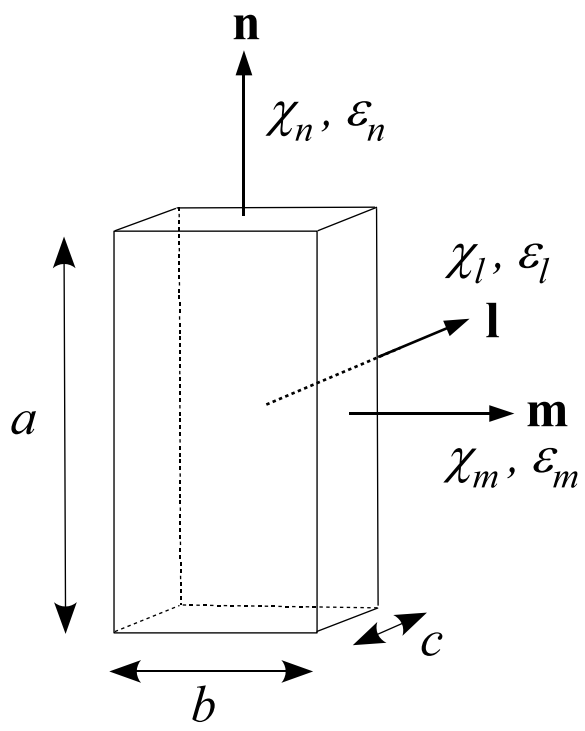

Figure 1: A schematic diagram for the mathematical description of the biaxial phase via a biaxial plate, not to be confused with a biaxial molecule. The major director $\mathbf{n}$ and the minor director $\mathbf{m}$ are as indicated for dimensions $a>b>c$, with $\mathbf{l}=\mathbf{n} \times \mathbf{m}$. For each axis of the biaxial plate there is an associated magnetic susceptibility $\chi_{i}$ and dielectric permittivity $\epsilon_{i}$, which correspond to the principal components of the associated tensors.

the vector $\mathbf{n}$ will correspond to the usual idea of the 'long' uniaxial director of nematic liquid crystals and the vector $\mathbf{m}$ will be referred to as the minor director; $\mathbf{n}$ and $\mathbf{m}$ are also referred to as the major and minor directors, respectively. The rotation of the biaxial plate around the direction of the major director $\mathbf{n}$ is uniquely determined by the orientation of $\mathbf{m}$ while the unit vector $\mathbf{l}$ is simply determined from the relation $\mathbf{l}=\mathbf{n} \times \mathbf{m}$ and thus knowledge of the orientation of $\mathbf{n}$ and $\mathbf{m}$ is sufficient to describe the biaxial alignment, with $\mathbf{l}$ being chiefly employed to ease notation and identify the third axis of symmetry. The mathematical constraints on the two essential directors $\mathbf{n}(\mathbf{x}, t)$ and $\mathbf{m}(\mathbf{x}, t)$ are therefore

$$
\mathbf{n} \cdot \mathbf{n}=\mathbf{m} \cdot \mathbf{m}=1, \quad \mathbf{n} \cdot \mathbf{m}=0 .
$$

In static problems it is common to minimise, via suitable Euler-Lagrange equations, an energy based upon the possible distortions of the directors $\mathbf{n}$ and $\mathbf{m}$. This energy typically involves contributions from elastic, magnetic and electric energies, which will now be summarised briefly. The total energy, $W$, is given by

$$
W(\mathbf{n}, \mathbf{m}, \nabla \mathbf{n}, \nabla \mathbf{m})=\int_{V}\left\{w_{e l}(\mathbf{n}, \mathbf{m}, \nabla \mathbf{n}, \nabla \mathbf{m})+w_{m}(\mathbf{n}, \mathbf{m})+w_{e}(\mathbf{n}, \mathbf{m})\right\} d V,
$$

where $V$ is the sample volume and $w_{e l}, w_{m}$ and $w_{e}$ are the elastic, magnetic and electric energy densities, respectively. Various possible symmetries and alignments of differing biaxial nematics have been discussed by Karahaliou et al. [28]. However, the material 
symmetries that will be supposed in this rudimentary discussion will be based on those selected by Leslie et al. [16] who, by extending the usual uniaxial symmetry of nematics, imposed the requirements

$$
W(\mathbf{n}, \mathbf{m}, \nabla \mathbf{n}, \nabla \mathbf{m})=W(-\mathbf{n}, \mathbf{m},-\nabla \mathbf{n}, \nabla \mathbf{m})=W(\mathbf{n},-\mathbf{m}, \nabla \mathbf{n},-\nabla \mathbf{m}) .
$$

The energy density must also be frame-indifferent and is therefore subject to the usual invariance to arbitrary superposed rigid body rotations.

\subsection{The elastic energy}

Frame-indifference, in conjunction with the symmetry requirement (2.3), leads to the elastic energy density for a biaxial nematic. The most general form that is quadratic in the gradients of the directors that meets these conditions has been derived by Govers and Vertogen [12] and can be written as

$$
\begin{aligned}
w_{e l}(\mathbf{n}, \mathbf{m}, \nabla \mathbf{n}, \nabla \mathbf{m})= & \frac{1}{2} K_{1}(\nabla \cdot \mathbf{n})^{2}+\frac{1}{2} K_{2}(\mathbf{n} \cdot \nabla \times \mathbf{n})^{2}+\frac{1}{2} K_{3}(\mathbf{n} \times \nabla \times \mathbf{n})^{2} \\
& +\frac{1}{2} K_{4}(\nabla \cdot \mathbf{m})^{2}+\frac{1}{2} K_{5}(\mathbf{m} \cdot \nabla \times \mathbf{m})^{2}+\frac{1}{2} K_{6}(\mathbf{m} \times \nabla \times \mathbf{m})^{2} \\
& +\frac{1}{2} K_{7}[\mathbf{n} \cdot(\mathbf{m} \times \nabla \times \mathbf{m})]^{2}+\frac{1}{2} K_{8}[\mathbf{m} \cdot(\mathbf{n} \times \nabla \times \mathbf{n})]^{2} \\
& +\frac{1}{2} K_{9}[\mathbf{m} \cdot \nabla \times(\mathbf{n} \times \mathbf{m})]^{2}+\frac{1}{2} K_{10}[\mathbf{n} \cdot \nabla \times(\mathbf{m} \times \mathbf{n})]^{2} \\
& +\frac{1}{2} K_{11}[\nabla \times(\mathbf{n} \times \mathbf{m})]^{2}+\frac{1}{2} K_{12}[\nabla \cdot(\mathbf{n} \times \mathbf{m})]^{2},
\end{aligned}
$$

where the $K_{i}, i=1,2, \ldots 12$, are elastic constants. The uniaxial nematic energy density, expressible in terms of the standard splay, twist and bend elastic constants, $K_{1}, K_{2}$ and $K_{3}$, respectively, can be recovered from this energy density by eliminating any contributions that contain the minor director $\mathbf{m}$.

If a particular biaxial nematic liquid crystal happens to be chiral then three additional twist terms [13] that are linear in gradients must be added to the elastic energy $w_{e l}$ given in equation (2.4) so that it may be replaced by $w_{e l}+w_{c}$ where the additional energy density $w_{c}$ is given by

$$
w_{c}=k_{1}(\mathbf{n} \cdot \nabla \times \mathbf{n})+k_{2}(\mathbf{m} \cdot \nabla \times \mathbf{m})+k_{3}(\mathbf{l} \cdot \nabla \times \mathbf{l}),
$$

where $k_{1}, k_{2}$ and $k_{3}$ are twist elastic constants.

\subsection{The magnetic and electric energies}

To describe the magnetic and electric properties of biaxial nematics it is necessary to introduce three magnetic susceptibilities and three dielectric permittivities, corresponding to each principal axis of the biaxial plate as shown in Fig. 1 (see also Chapter 4). The magnetic susceptibility along the principal $i$-axis is labelled $\chi_{i}$, and similarly for the dielectric permittivity $\epsilon_{i}$, where $i$ takes any one of the symbols $n, m$ or $l$ as appropriate. Analogous to the continuum theory for nematics $[29,30]$, it is also convenient to introduce the corresponding magnetic and dielectric anisotropies

$$
\chi_{i j}=\chi_{i}-\chi_{j}, \quad \epsilon_{i j}=\epsilon_{i}-\epsilon_{j} .
$$


The application of a magnetic field $\mathbf{H}$ to a biaxial nematic liquid crystal leads to an induced magnetisation $\mathbf{M}$ given by $[16,27]$

$$
\mathbf{M}=\mu_{0}\left[\chi_{n}(\mathbf{H} \cdot \mathbf{n}) \mathbf{n}+\chi_{m}(\mathbf{H} \cdot \mathbf{m}) \mathbf{m}+\chi_{l}(\mathbf{H} \cdot \mathbf{l}) \mathbf{l}\right]
$$

where $\mu_{0}$ is the permeability of free space. By an application of the identity [12]

$$
\delta_{i j}=n_{i} n_{j}+m_{i} m_{j}+l_{i} l_{j}
$$

where $\delta_{i j}$ is the usual Kronecker delta, the products of the components of the vector $\mathbf{l}$ can be expressed in terms of the components of $\mathbf{n}$ and $\mathbf{m}$. The notation in eq.(2.6) then allows the magnetisation to be rewritten in terms of $\mathbf{n}$ and $\mathbf{m}$ only via a straightforward substitution for $\mathbf{l}$, namely,

$$
\mathbf{M}=\mu_{0}\left[\chi_{n l}(\mathbf{H} \cdot \mathbf{n}) \mathbf{n}+\chi_{m l}(\mathbf{H} \cdot \mathbf{m}) \mathbf{m}+\chi_{l} \mathbf{H}\right] .
$$

We remark here that since the magnetic induction $\mathbf{B}$ satisfies the relation

$$
\mathbf{B}=\mu_{0}(\mathbf{H}+\mathbf{M})
$$

an alternative form [27] for the magnetisation in terms of $\mathbf{B}$ can be derived provided the magnetic susceptibilities are small, namely,

$$
\mathbf{M}=\mu_{0}^{-1}\left[\chi_{n l}(\mathbf{B} \cdot \mathbf{n}) \mathbf{n}+\chi_{m l}(\mathbf{B} \cdot \mathbf{m}) \mathbf{m}+\chi_{l} \mathbf{B}\right]
$$

analogous to the situation for nematics [30,31]. The magnetic energy density can be shown to be $[27,29]$

$$
w_{m}=-\int_{\mathbf{0}}^{\mathbf{H}} \mathbf{M} \cdot d \mathbf{H}=-\frac{1}{2} \mathbf{M} \cdot \mathbf{H},
$$

and therefore the magnetic energy density can be obtained from eq.(2.9) as

$$
w_{m}=-\frac{1}{2} \mu_{0}\left[\chi_{n l}(\mathbf{H} \cdot \mathbf{n})^{2}+\chi_{m l}(\mathbf{H} \cdot \mathbf{m})^{2}+\chi_{l} H^{2}\right],
$$

where $H=|\mathbf{H}|$ is the magnitude of the field. Notice that the last term that involves $\chi_{l}$ in eq.(2.13) is independent of the orientation of both the directors $\mathbf{n}$ and $\mathbf{m}$. In terms of the magnetic induction the corresponding energy density is analogously given by

$$
w_{m}=-\frac{1}{2} \mu_{0}^{-1}\left[\chi_{n l}(\mathbf{B} \cdot \mathbf{n})^{2}+\chi_{m l}(\mathbf{B} \cdot \mathbf{m})^{2}+\chi_{l} B^{2}\right]
$$

where $B=|\mathbf{B}|$.

As discussed by Ericksen [32], the magnetisation $\mathbf{M}$ leads to a body force $\rho \mathbf{F}$ and moment $\rho \mathbf{K}$ given by, respectively,

$$
\rho F_{i}=M_{j} H_{j, i}, \quad \rho K_{i}=\epsilon_{i j k} M_{j} H_{k}
$$

where $\rho$ is the density and $\epsilon_{i j k}$ is the usual alternator. The moment may be expressed as $[16]$

$$
\rho K_{i}=\epsilon_{i j k}\left(n_{j} G_{k}^{n}+m_{j} G_{k}^{m}\right),
$$

where the generalised torques $\mathbf{G}^{n}$ and $\mathbf{G}^{m}$ can be introduced as

$$
G_{i}^{n}=\mu_{0} \chi_{n l} n_{j} H_{j} H_{i}, \quad G_{i}^{m}=\mu_{0} \chi_{m l} m_{j} H_{j} H_{i} .
$$


It is evident that if the magnetic potential is introduced as $\Psi_{m} \equiv-w_{m}$ then, analogous to the situation for nematic liquid crystals [30, 4.2 .4$]$,

$$
\rho F_{i}=\frac{\partial \Psi_{m}}{\partial x_{i}}, \quad G_{i}^{n}=\frac{\partial \Psi_{m}}{\partial n_{i}}, \quad G_{i}^{m}=\frac{\partial \Psi_{m}}{\partial m_{i}},
$$

and so

$$
\rho F_{i}+G_{j}^{n} n_{j, i}+G_{j}^{m} m_{j, i}=\Psi_{m, i},
$$

a result that can simplify calculations (see, for example, Section 4.1). These director expressions are of particular importance when modelling director dynamics.

An expression for the induced electric displacement $\mathbf{D}$ is analogous to that for the magnetic field and may be written as

$$
\mathbf{D}=\epsilon_{0}\left[\epsilon_{n l}(\mathbf{E} \cdot \mathbf{n}) \mathbf{n}+\epsilon_{m l}(\mathbf{E} \cdot \mathbf{m}) \mathbf{m}+\epsilon_{l} \mathbf{E}\right],
$$

where $\epsilon_{0}$ is the permittivity of free space. The consequent electric energy density is then

$$
w_{e}=-\frac{1}{2} \epsilon_{0}\left[\epsilon_{n l}(\mathbf{E} \cdot \mathbf{n})^{2}+\epsilon_{m l}(\mathbf{E} \cdot \mathbf{m})^{2}+\epsilon_{l} E^{2}\right],
$$

where $E=|\mathbf{E}|$. The results in equations (2.15) to (2.19) for a magnetic field have their obvious analogues for the electric field case where the electric potential $\Psi_{e} \equiv-w_{e}$ can be introduced. We remark that it is also possible to incorporate the effect of gravity as a body force in a similar way via the gravitational potential [30].

\subsection{The total energy}

The energy contributions that appear in the total energy density will depend on what is considered to be of primary importance in any appropriate model problem; this is open to a number of factors and simplifying assumptions. In general, the total energy for a biaxial nematic liquid crystal under the influence of an electromagnetic field may be expressed as

$$
W=\int_{V} w d V, \quad \text { where } \quad w=w_{e l}+w_{m}+w_{e},
$$

and $w_{e l}, w_{m}$ and $w_{e}$ are given by eqs.(2.4), (2.13) and (2.21), respectively; $w_{m}$ may be replaced by the alternative form in (2.14) if required. Any number of these energy densities may be included or omitted in the preliminary stages of a modelling problem; frequently, simplifying assumptions on possible distortions and influences may considerably reduce the complexity of these contributory energy densities in basic model problems. The forms for the generalised torques expressed in eq.(2.17) are widely used in dynamics and static equilibrium situations. For problems that involve dynamics it is often more convenient to separate the elastic energy $w_{e l}$ from the total energy and consider it in conjunction with the potential $\Psi=\Psi_{m}+\Psi_{e}$ in an alternative formulation.

\section{Dynamic equations}

Before summarising the continuum equations developed by Leslie et al. [16] in section 3.3, it is necessary to introduce some standard results on the continuum balance laws and viscous stress for incompressible biaxial liquid crystals. 


\subsection{Balance laws}

A superposed dot, or the notation $D / D t$, represents the usual material time derivative given by

$$
\frac{D}{D t}=\frac{\partial}{\partial t}+\mathbf{v} \cdot \frac{\partial}{\partial \mathbf{x}}
$$

where $\mathbf{x}$ is the position vector and $\mathbf{v}$ is the velocity of the fluid. Note that this operator may be applied to a scalar or vector quantity. For a volume $V$ of liquid crystal bounded by the surface $S$ the three conservation laws for mass, linear momentum and angular momentum are, respectively,

$$
\begin{aligned}
\frac{D}{D t} \int_{V} \rho d V & =0 \\
\frac{D}{D t} \int_{V} \rho \mathbf{v} d V & =\int_{V} \rho \mathbf{F} d V+\int_{S} \mathbf{t} d S, \\
\frac{D}{D t} \int_{V} \rho(\mathbf{x} \times \mathbf{v}) d V & =\int_{V} \rho(\mathbf{x} \times \mathbf{F}+\mathbf{K}) d V+\int_{S}(\mathbf{x} \times \mathbf{t}+\mathbf{l}) d S,
\end{aligned}
$$

where $\mathbf{F}$ is the external body force per unit mass, $\mathbf{t}$ is the surface force per unit area, $\mathbf{K}$ is the external body moment per unit mass and $\mathbf{l}$ is the surface moment per unit area (also called the couple stress vector). It is well known that the components $t_{i}$ and $l_{i}$ of the surface force and surface moment are expressible in terms of the stress tensor $t_{i j}$ and couple stress tensor $l_{i j}$ through the relations

$$
t_{i}=t_{i j} \nu_{j}, \quad l_{i}=l_{i j} \nu_{j},
$$

where $\boldsymbol{\nu}$ is the outward unit normal to the surface $S$.

If the sample is assumed to be incompressible then standard results show that the three balance laws eqs. $(3.2-3.4)$ can be reduced to the point forms

$$
\begin{aligned}
v_{i, i} & =0, \\
\rho \dot{v}_{i} & =\rho F_{i}+t_{i j, j}, \\
0 & =\rho K_{i}+\epsilon_{i j k} t_{k j}+l_{i j, j} .
\end{aligned}
$$

We denote the local angular velocity of the liquid crystal material element by $\mathbf{w}$ and let $\mathcal{D}$ be the rate of viscous dissipation per unit volume. As mentioned at the end of the previous section, it is often convenient in dynamics to consider $w_{e l}$ as the elastic energy density and introduce the magnetic or electric fields via the generalised torques using potentials, such as those in eq.(2.17). A virtual work hypothesis is supposed of the form [16]

$$
\int_{V} \rho(\mathbf{F} \cdot \mathbf{v}+\mathbf{K} \cdot \mathbf{w}) d V+\int_{S}(\mathbf{t} \cdot \mathbf{v}+\mathbf{l} \cdot \mathbf{w}) d S=\frac{D}{D t} \int_{V}\left(\frac{1}{2} \rho \mathbf{v} \cdot \mathbf{v}+w_{e l}\right) d V+\int_{V} \mathcal{D} d V .
$$

This is identical in form to that proposed for nematic liquid crystals by Leslie [33] when he presented a simplified derivation of the continuum theory of uniaxial nematics in 1992; similar postulates can be found in the theories of polar materials $[34,35]$ and in the theory of smectic $\mathrm{C}$ liquid crystals [30]. Using the results in eqs.(3.7) and (3.8) and Reynolds' transport theorem allows this postulate to be given in point form as

$$
t_{i j} v_{i, j}+l_{i j} w_{i, j}-w_{i} \epsilon_{i j k} t_{k j}=\dot{w}_{e l}+\mathcal{D} \text {. }
$$


Since $\mathbf{w}$ is the local angular velocity of the material element, that is, it represents the local angular velocity of both of the directors, it follows that [16]

$$
\dot{\mathbf{n}}=\mathbf{w} \times \mathbf{n}, \quad \dot{\mathbf{m}}=\mathbf{w} \times \mathbf{m},
$$

because $\mathbf{n}$ and $\mathbf{m}$ are unit vectors. These results, coupled with the symmetry conditions on $w_{e l}$, allow the relation (3.10) to be exploited: since the viscous dissipation is necessarily positive, detailed calculations reveal that [16]

$$
\begin{aligned}
t_{i j} & =-p \delta_{i j}-\frac{\partial w_{e l}}{\partial n_{p, j}} n_{p, i}-\frac{\partial w_{e l}}{\partial m_{p, j}} m_{p, i}+\tilde{t}_{i j} \\
l_{i j} & =\epsilon_{i p q}\left(n_{p} \frac{\partial w_{e l}}{\partial n_{q, j}}+m_{p} \frac{\partial w_{e l}}{\partial m_{q, j}}\right)+\tilde{l}_{i j}
\end{aligned}
$$

where $p$ is an arbitrary pressure resulting from the assumed incompressibility and $\tilde{t}_{i j}$ and $\tilde{l}_{i j}$ denote possible dynamic contributions. In the general terminology of Leslie, $\tilde{t}_{i j}$ is called the viscous stress (cf. [36, p.36]). This further reduces the relationship eq.(3.10) to

$$
\tilde{t}_{i j} v_{i, j}+\tilde{l}_{i j} w_{i, j}-w_{i} \epsilon_{i j k} \tilde{t}_{k j}=\mathcal{D} \geq 0,
$$

given that $\mathcal{D}$ is positive. This inequality is of crucial importance when investigating the constitutive theory of biaxial nematics and it imposes restrictions upon the forms of the dynamic contributions. For example, if there is no supposed dependence upon the derivatives of the local angular velocity $\mathbf{w}$ then it follows immediately that

$$
\tilde{l}_{i j}=0,
$$

so that eq.(3.14) simplifies to

$$
\tilde{t}_{i j} v_{i, j}-w_{i} \epsilon_{i j k} \tilde{t}_{k j}=\mathcal{D} \geq 0 .
$$

The rate of strain tensor $A$ and vorticity tensor $W$ are second order tensors defined in the usual way, in an obvious nomenclature, by

$$
\mathrm{A}=\frac{1}{2}\left(\nabla \mathbf{v}+(\nabla \mathbf{v})^{\mathrm{T}}\right), \quad \mathrm{W}=\frac{1}{2}\left(\nabla \mathbf{v}-(\nabla \mathbf{v})^{\mathrm{T}}\right) .
$$

Notice that $A$ is symmetric and $W$ is skew-symmetric. Following Leslie et al. [16], we introduce the co-rotational time flux vectors of $\mathbf{n}$ and $\mathbf{m}$ defined respectively by

$$
\mathbf{N}=\dot{\mathbf{n}}-\mathbf{W n}, \quad \mathbf{M}=\dot{\mathbf{m}}-W_{\mathbf{m}},
$$

these being analogous to the term discussed originally by Ericksen and Leslie for uniaxial nematics. The vector $\mathbf{M}$ in this expression is not to be confused with the magnetisation introduced earlier: the context will make this clear. It is worth noting that

$$
\mathbf{M} \cdot \mathbf{n}+\mathbf{N} \cdot \mathbf{m}=0
$$

because $\mathbf{W}$ is skew-symmetric and $\mathbf{n}$ and $\mathbf{m}$ are mutually orthogonal.

In liquid crystals it is important to distinguish between different types of angular velocity [30]. The local angular velocity $\mathbf{w}$ of the material element has been introduced 
above. The only independent field in the continuum theory of isotropic fluids is the velocity $\mathbf{v}$ and it is well known that the corresponding angular velocity for such fluids, denoted by $\widehat{\mathbf{w}}$, is one half of the curl of the velocity, that is,

$$
\widehat{\mathbf{w}}=\frac{1}{2} \nabla \times \mathbf{v} .
$$

This particular angular velocity is called the regional angular velocity, to distinguish it from other angular velocities. The angular velocity of the material element relative to the regional angular velocity in which the material element is embedded is denoted by $\boldsymbol{\omega}$ and is defined by $[16,30]$

$$
\boldsymbol{\omega}=\mathbf{w}-\widehat{\mathbf{w}}=\mathbf{w}-\frac{1}{2} \nabla \times \mathbf{v} .
$$

The quantity $\boldsymbol{\omega}$ is called the relative angular velocity and is introduced to measure the difference between the local angular velocity $\mathbf{w}$ of a liquid crystal director and the regional angular velocity $\widehat{\mathbf{w}}$ of the fluid in the neighbourhood of such a director. It can be shown (cf. [30, p.135]) that

$$
\mathbf{N}=\boldsymbol{\omega} \times \mathbf{n} \quad \text { and } \quad \mathbf{M}=\boldsymbol{\omega} \times \mathbf{m} .
$$

The quantities $\mathbf{N}$ and $\mathbf{M}$, therefore, measure the rotation of $\mathbf{n}$ and $\mathbf{m}$ relative to the rotation of the fluid. We can verify by calculation that $\boldsymbol{\omega}$ may be expressed as [16]

$$
\boldsymbol{\omega}=\mathbf{n} \times \mathbf{N}+\mathbf{m} \times \mathbf{M}+(\mathbf{m} \cdot \mathbf{N})(\mathbf{m} \times \mathbf{n}),
$$

which is a consequence of the identity stated in eq.(2.8), and that $\mathbf{w}$ has the representation $[25]$

$$
2 \mathbf{w}=\mathbf{n} \times \dot{\mathbf{n}}+\mathbf{m} \times \dot{\mathbf{m}}+\mathbf{l} \times \dot{\mathbf{l}} .
$$

\subsection{The viscous stress}

Symmetry conditions dictate that $\tilde{t}_{i j}$ is an isotropic function of $n_{i}, m_{i}, N_{i}, M_{i}$ and $A_{i j}$, being even in both $\mathbf{n}$ and $\mathbf{m}$. Under the assumption that the viscous stress is linear in $\mathbf{N}, \mathbf{M}$ and the velocity gradients, the viscous stress is revealed to be $[16,17,24]$

$$
\begin{aligned}
\tilde{t}_{i j}= & \alpha_{1} n_{k} A_{k p} n_{p} n_{i} n_{j}+\alpha_{2} N_{i} n_{j}+\alpha_{3} N_{j} n_{i}+\alpha_{4} A_{i j}+\alpha_{5} n_{j} A_{i k} n_{k}+\alpha_{6} n_{i} A_{j k} n_{k} \\
& +\beta_{1} m_{k} A_{k p} m_{p} m_{i} m_{j}+\beta_{2} M_{i} m_{j}+\beta_{3} M_{j} m_{i}+\beta_{5} m_{j} A_{i k} m_{k}+\beta_{6} m_{i} A_{j k} m_{k} \\
& +\left(\mu_{1} m_{i} n_{j}+\mu_{2} m_{j} n_{i}\right) N_{p} m_{p}+\left(\mu_{3} m_{i} n_{j}+\mu_{4} m_{j} n_{i}\right) n_{k} A_{k p} m_{p} \\
& +\mu_{5} m_{k} A_{k p} m_{p} n_{i} n_{j}
\end{aligned}
$$

where the $\alpha_{i}, \beta_{i}$ and $\mu_{i}$ are dynamic viscosity coefficients. It proves convenient to introduce dynamic contributions $\tilde{\mathbf{g}}^{n}$ and $\tilde{\mathbf{g}}^{m}$ that are associated with the asymmetric part of the viscous stress $[16,24]$ through the formulation

$$
\epsilon_{i j k} \tilde{t}_{k j}=\epsilon_{i j k}\left(n_{j} \tilde{g}_{k}^{n}+m_{j} \tilde{g}_{k}^{m}\right) .
$$

They are given by

$$
\begin{aligned}
\tilde{g}_{i}^{n} & =-\left(\gamma_{1} N_{i}+\gamma_{2} A_{i j} n_{j}+\gamma_{3} N_{j} m_{j} m_{i}+\gamma_{4} n_{j} A_{j k} m_{k} m_{i}\right) \\
\tilde{g}_{i}^{m} & =-\left(\lambda_{1} M_{i}+\lambda_{2} A_{i j} m_{j}\right)
\end{aligned}
$$


where

$$
\begin{array}{lll}
\gamma_{1}=\alpha_{3}-\alpha_{2}, & \gamma_{2}=\alpha_{6}-\alpha_{5}, & \gamma_{3}=\mu_{2}-\mu_{1}, \\
\gamma_{4}=\mu_{4}-\mu_{3}, & \lambda_{1}=\beta_{3}-\beta_{2}, & \lambda_{2}=\beta_{6}-\beta_{5} .
\end{array}
$$

There are sixteen viscosity coefficients in this formulation. However, if Onsager symmetry relations are adopted, as is common in liquid crystal theories [30], then it can be shown that $[16,24]$

$$
\gamma_{2}=\alpha_{2}+\alpha_{3}, \quad \lambda_{2}=\beta_{2}+\beta_{3}, \quad \gamma_{4}=\mu_{1}+\mu_{2}, \quad \mu_{5}=0,
$$

which reduces the number of independent viscosity coefficients to twelve. This reduction also ensures that the viscous stress tensor is derivable from a dissipation function: details can be found in [16]. When the relations eqs.(3.30) are adopted then the viscous stress reduces to the same number of terms discussed earlier in the literature by Saupe [9] and Govers and Vertogen [14]. The viscous stress for uniaxial nematics can be recovered from this form of $\tilde{t}_{i j}$ by setting all the $\beta_{i}$ and $\mu_{i}$ coefficients in eq.(3.25) to zero.

An interpretation of the viscous stress, as given by Carlsson et al. [26], can be made in the context of Fig. 1. The six $\alpha_{i}$ terms, which are the ones acting on the major director $\mathbf{n}$, correspond exactly to the terms in the viscous stress tensor for uniaxial nematics $[37,38]$. Note that the $\alpha_{4}$ terms corresponds to the usual isotropic Newtonian viscosity $\eta$ through the relation $\eta \equiv \frac{1}{2} \alpha_{4}$. The five $\beta_{i}$ terms are those acting on the minor director $\mathbf{m}$ and they have exactly the same structure as the corresponding five $\alpha_{i}$ terms except that $\mathbf{n}$ is replaced by $\mathbf{m}$. The $\mu_{i}$ terms reflect the coupling effects between the major and minor directors; these particular terms have no counterparts in the theory of uniaxial nematics.

Some speculative theoretical observations made by Carlsson et al. [25] should be recorded here as a guide to the magnitudes of the viscosity coefficients so that theoretical estimates may be made which are realistic and guided by what is known from the extensive literature on uniaxial nematics. With reference to the geometry in Fig. 1, in the case where $a>b>c$ it is natural to suppose that the major director $\mathbf{n}$ is more dominant than the minor director $\mathbf{m}$. It is therefore expected that the $\alpha_{i}$ coefficients ought to be of the same order of magnitude as the established corresponding Leslie viscosities $[30,39]$ of uniaxial nematics, also labelled $\alpha_{i}$. The five $\beta_{i}$ coefficients should have a similar relationship to the viscous behaviour of the material as experienced by the correspondingly indexed $\alpha_{i}$ coefficients; if $a \gg b$ then the $\beta_{i}$ should additionally be much smaller in magnitude than their $\alpha_{i}$ counterparts. Although the $\beta_{i}$ may be smaller than the $\alpha_{i}$, Carlsson et al. [25] have argued that the $\mu_{i}$ coefficients cannot be significantly smaller in magnitude than the $\beta_{i}$ coefficients. The case when $\alpha_{i}=\beta_{i}$ corresponds to setting $a=b>c$ in Fig. 1 and this leads to the study of a disk-like nematic where the effective director becomes $\mathbf{l}$ and the viscous stress reduces to seven terms (see [17] for details). Similarly, if $a \gtrsim b \gg c$ then we again have a situation which corresponds to a disk-like nematic. In summary, if $a>b>c$ then is is reasonable to assume that $\left|\alpha_{i}\right|>\left|\beta_{i}\right|$, with the $\beta_{i}$ having the same approximate magnitudes as the $\mu_{i}$, and that if $a \gg b>c$ then $\left|\alpha_{i}\right| \gg\left|\beta_{i}\right|$ with, as before, the $\beta_{i}$ similar in magnitude to the $\mu_{i}$.

Some inequalities regarding the viscosity coefficients have been derived by Leslie et al. [16] by means of a dissipation inequality when the relations eq.(3.30) hold, that is, 
when there are twelve independent viscosities. These inequalities, combined with the comments in the previous paragraph, may act as guides to appropriate estimates for theoretical work and modelling to data when many of the viscosities are unknown for particular materials. They are

$$
\begin{gathered}
2 \alpha_{4}+\alpha_{5}+\alpha_{6}>0, \quad 2 \alpha_{4}+\beta_{5}+\beta_{6}>0 \\
2 \alpha_{4}+\alpha_{5}+\alpha_{6}+\beta_{5}+\beta_{6}+\mu_{3}+\mu_{4}>0, \quad 2 \alpha_{4}+\alpha_{5}+\alpha_{6}+\alpha_{1}>0 \\
2 \alpha_{4}+\beta_{5}+\beta_{6}+\beta_{1}>0, \quad \gamma_{1}>0, \quad \lambda_{1}>0, \quad \gamma_{1}+\lambda_{1}+\gamma_{3}>0 \\
\alpha_{4}^{2}<\left(2 \alpha_{4}+\alpha_{5}+\alpha_{6}+\alpha_{1}\right)\left(2 \alpha_{4}+\beta_{5}+\beta_{6}+\beta_{1}\right) \\
\gamma_{2}^{2}<\gamma_{1}\left(2 \alpha_{4}+\alpha_{5}+\alpha_{6}\right), \quad \lambda_{2}^{2}<\lambda_{1}\left(2 \alpha_{4}+\beta_{5}+\beta_{6}\right) \\
\left(\gamma_{2}-\lambda_{2}+\gamma_{4}\right)^{2}<\left(\gamma_{1}+\lambda_{1}+\gamma_{3}\right)\left(2 \alpha_{4}+\alpha_{5}+\alpha_{6}+\beta_{5}+\beta_{6}+\mu_{3}+\mu_{4}\right) .
\end{gathered}
$$

Carlsson et al. [25] have shown that three rotational viscosities and nine effective shearing viscosities can be defined in order to completely characterise the viscous behaviour of a biaxial nematic when eq.(3.30) holds, that is, when the number of viscosities can be reduced to twelve. Of these twelve viscosities listed by these authors, the most crucial in elementary descriptions are the three key (positive) rotational viscosities: they have been identified, via a priori positivity requirements, as

$$
\begin{aligned}
\gamma_{n} & =\alpha_{3}-\alpha_{2}>0, \\
\gamma_{m} & =\beta_{3}-\beta_{2}>0, \\
\gamma_{n m} & =\alpha_{3}-\alpha_{2}+\beta_{3}-\beta_{2}+\mu_{2}-\mu_{1}>0 .
\end{aligned}
$$

Notice that these inequalities are included in eq.(3.31) and can be written, in terms of the notation introduced in eq.(3.29), as

$$
\gamma_{n} \equiv \gamma_{1}, \quad \gamma_{m} \equiv \lambda_{1}, \quad \gamma_{n m} \equiv \gamma_{n}+\gamma_{m}+\gamma_{3}
$$

Each of these viscosities is related to a rotation around one of the principal axes of the biaxial plate, as shown in Fig. 2, and this alternative nomenclature has been introduced for notational convenience in order to highlight their influence relative to the directors. In Fig. 2(a) the minor director $\mathbf{m}$ rotates around the axis of the major director $\mathbf{n}$ and
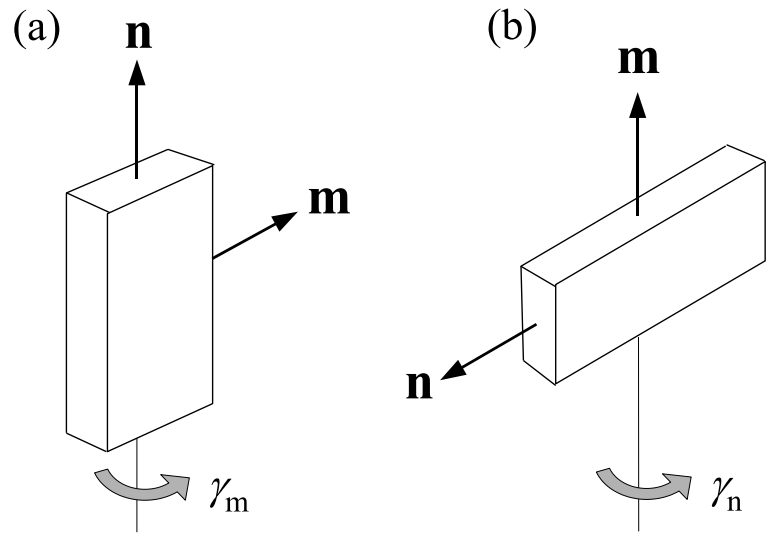

(c)

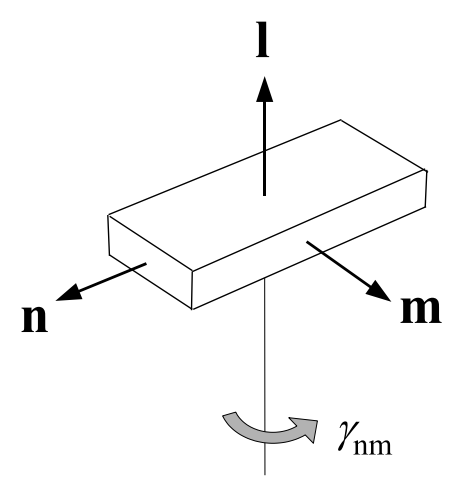

Figure 2: The three key rotational viscosities of a biaxial nematic. 
this viscous effect has a related rotational viscosity $\gamma_{m}$ due to the local rotation of $\mathbf{m}$. Similarly, in Fig. 2(b) the major director $\mathbf{n}$ rotates around the axis of the minor director $\mathbf{m}$ and this rotation is related to the rotational viscosity $\gamma_{n}$. The two rotational viscosities $\gamma_{n}$ and $\gamma_{m}$ therefore correspond to the situations for which only one of the two directors $\mathbf{n}$ and $\mathbf{m}$ rotates. The structure of these viscosities emphasises the aforementioned comments that the $\beta_{i}$ coefficients are related to the director $\mathbf{m}$ in the same way that the $\alpha_{i}$ coefficients are related to $\mathbf{n}$. The rotational viscosity $\gamma_{n}$ is familiar from the classical theory of nematics $[29,30]$. The situation in which both directors $\mathbf{n}$ and $\mathbf{m}$ rotate around the axis $\mathbf{l}$, as shown in Fig. 2(c), is linked to the rotational viscosity $\gamma_{n m}$. We remark that $\gamma_{n m}=\gamma_{n}+\gamma_{m}+\gamma_{3}$, which shows that this particular viscosity is the sum of the two independent rotational viscosities $\gamma_{n}$ and $\gamma_{m}$ plus the contribution $\gamma_{3}=\mu_{2}-\mu_{1}$. This additional contribution represents a coupling between the rotations of the two directors $\mathbf{n}$ and $\mathbf{m}$. Carlsson et al. [25] have identified various equilibrium orientations of a biaxial alignment that result under shear flow, the consequent orientation angles of the directors being given in terms of the viscosities. The reader is referred to [25] for further details of these alignments and for a brief analysis of their stability in terms of the related effective viscosities; more extensive details on their stability, including evidence of bistability in the presence of electric and magnetic fields, can be found in $[26,27]$.

\subsection{The dynamic equations}

We are now in a position to summarise the continuum equations for the dynamics of an incompressible biaxial nematic liquid crystal. The full derivation can be found in [16].

We shall consider the elastic energy density $w_{e l}$ and allow the magnetic or electric field contributions to enter via the generalised torques $\mathbf{G}^{n}$ and $\mathbf{G}^{m}$ of the form introduced previously in eqs.(2.16) to (2.19), suitably adapted to cover the case of combined electric and magnetic fields, if required. The constraints on the major and minor directors lead to the requirements stated in eq.(2.1), namely,

$$
n_{i} n_{i}=1, \quad m_{i} m_{i}=1, \quad n_{i} m_{i}=0 .
$$

The results from eqs.(3.12) to (3.15) can be inserted into the balance laws given in eqs.(3.6) to (3.8) in order to arrive at the final continuum equations, making use of the generalised torques formulation. After quite detailed manipulations of these equations, the three main balance laws lead to the remaining governing equations. The first of these, resulting from the conservation of mass, leads to the classical incompressible flow condition

$$
v_{i, i}=0 .
$$

The balance law for linear momentum can be formulated as, in the notation introduced in eqs.(3.25), (3.27) and (3.28) for $\tilde{t}_{i j}, \tilde{\mathbf{g}}^{n}$ and $\tilde{\mathbf{g}}^{m}$,

$$
\rho \dot{v}_{i}=\rho F_{i}-\left(p+w_{e l}\right)_{, i}+\tilde{g}_{j}^{n} n_{j, i}+\tilde{g}_{j}^{m} m_{j, i}+G_{j}^{n} n_{j, i}+G_{j}^{m} m_{j, i}+\tilde{t}_{i j, j},
$$

while the balance law for angular momentum leads to the two systems of coupled equa- 
tions given by

$$
\begin{aligned}
\left(\frac{\partial w_{e l}}{\partial n_{i, j}}\right)_{, j}-\frac{\partial w_{e l}}{\partial n_{i}}+\tilde{g}_{i}^{n}+G_{i}^{n} & =\gamma n_{i}+\kappa m_{i} \\
\left(\frac{\partial w_{e l}}{\partial m_{i, j}}\right)_{, j}-\frac{\partial w_{e l}}{\partial m_{i}}+\tilde{g}_{i}^{m}+G_{i}^{m} & =\tau m_{i}+\kappa n_{i} .
\end{aligned}
$$

In these expressions, $\gamma, \kappa$ and $\tau$ are Lagrange multipliers (scalar functions) that result from the three constraints in eq.(3.36). The scalar $\kappa$ couples the two sets of eqs.(3.39) and (3.40) and we remark that eq.(3.40) is similar in form to eq.(3.39). Eq.(3.39) differs from that for uniaxial nematics only through the addition of the $\kappa \mathbf{m}$ term (of course $w_{e l}$ would be replaced by the usual nematic elastic energy density, obtained by omitting all the contributions that involve $\mathbf{m}$ in eq. $(2.4))$.

Eqs.(3.36) to (3.40) form the complete set of continuum equations for incompressible biaxial liquid crystals. There are thirteen equations and this matches the number of unknowns that have to be determined. These unknowns consist of six from the components of $\mathbf{n}$ and $\mathbf{m}$, three from $\mathbf{v}$, one from the pressure $p$, and three from the multipliers $\gamma, \kappa$ and $\tau$.

In many applications, especially those that require the evaluation of body or surface forces, it is important to work with the full stress tensor for liquid crystals and so it is worth recording here that the stress tensor and couple stress tensor are, by eqs.(3.12), (3.13) and (3.15), given by

$$
\begin{aligned}
t_{i j} & =-p \delta_{i j}-\frac{\partial w_{e l}}{\partial n_{p, j}} n_{p, i}-\frac{\partial w_{e l}}{\partial m_{p, j}} m_{p, i}+\tilde{t}_{i j} \\
l_{i j} & =\epsilon_{i p q}\left(n_{p} \frac{\partial w_{e l}}{\partial n_{q, j}}+m_{p} \frac{\partial w_{e l}}{\partial m_{q, j}}\right) .
\end{aligned}
$$

\subsection{Euler angle description}

There are a number of different ways of introducing and defining Euler angles to describe more easily the orientation of the biaxial plate. For convenience, and for the removal of any ambiguity, we adopt a common classical definition of these angles that follows [40], as shown in Fig. 3. Consider a reference frame $O x y z$ and its reorientation around the same origin as shown in Fig. 3(b) to a new frame as indicated by $O x^{\prime} y^{\prime} z^{\prime}$ in the figure. To describe this reorientation we proceed as follows. Firstly, consider a positive rotation of $O x y z$ around the $z$-axis through the angle $\phi$. This will rotate $O x$ to $O x_{0}$ and $O y$ to $O y_{0}$, as indicated in Fig. 3(b); the direction along $O y_{0}$ is called the line of nodes and is labelled $O N$ for reference. Secondly, make a positive rotation around $O y_{0}$ through the angle $\theta$ so that $O z$ and $O x_{0}$ rotate into the positions $O z^{\prime}$ and $O x_{1}$, respectively. Finally, make a positive rotation around $O z^{\prime}$ through the angle $\psi$ to bring $O x_{1}$ and $O y_{0}$ into the positions $O x^{\prime}$ and $O y^{\prime}$, respectively, as shown in the figure. The rotations are applied in the order $\phi, \theta$ and $\psi$; the angles themselves are known as the Euler angles. An alternative labelling convention for the triple $(\phi, \theta, \psi)$ uses $(\alpha, \beta, \gamma)$. Notice that the $x y$-plane cuts the $x^{\prime} y^{\prime}$ plane along the line of nodes $O N$. If the Euler angles are given then the orientation of the primed axes can be located relative to the reference frame Oxyz. 
(a)

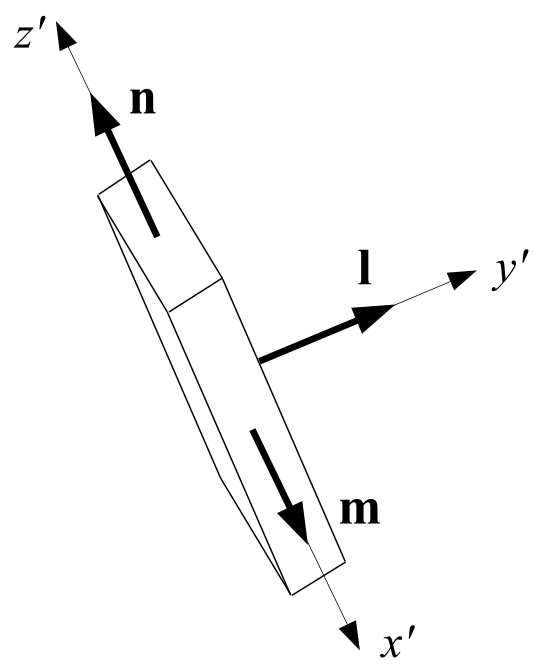

(b)

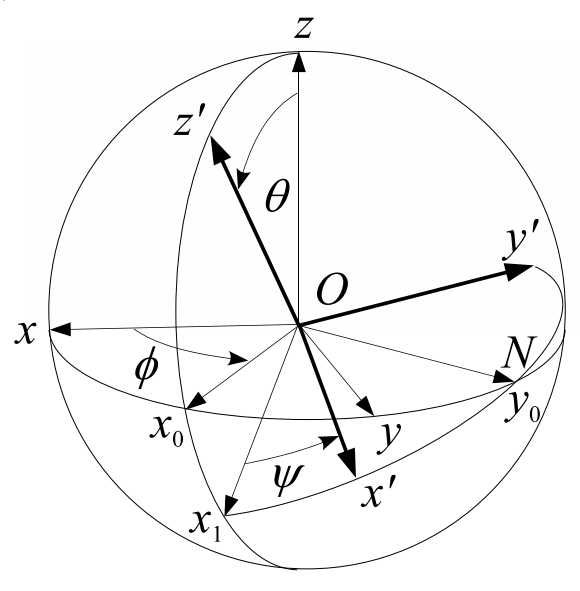

Figure 3: The orientation of a biaxial nematic liquid crystal described by using the standard Euler angles $\phi, \theta$ and $\psi$.

For example, the reference state of a biaxial plate may be given at $\phi=\theta=\psi \equiv 0$ with $\mathbf{n}$ and $\mathbf{m}$ pointing along the positive directions of the $z$-axis and $x$-axis, respectively. As the Euler angles change, the directors reorient according to the alignment of the corresponding axes in the $O x^{\prime} y^{\prime} z^{\prime}$ frame, with $\mathbf{n}$ and $\mathbf{m}$ aligned along the $z^{\prime}$-axis and $x^{\prime}$ axis, respectively. The angle $\psi$ always represents the rotation of the biaxial plate around the axis of the major director $\mathbf{n}$. The Euler angles, relative to the reference frame, for the biaxial plate shown in Fig. 3(a) coincide with those depicted in Fig. 3(b). Specifically, we can write the components of $\mathbf{n}$ and $\mathbf{m}$, in an obvious notation, as [40, p.261]

$$
n_{x}=\sin \theta \cos \phi, \quad n_{y}=\sin \theta \sin \phi, \quad n_{z}=\cos \theta,
$$

and

$$
\begin{aligned}
& m_{x}=-\sin \phi \sin \psi+\cos \theta \cos \phi \cos \psi, \\
& m_{y}=\cos \phi \sin \psi+\cos \theta \sin \phi \cos \psi, \\
& m_{z}=-\sin \theta \cos \psi .
\end{aligned}
$$

For completeness, we state that

$$
\begin{aligned}
& l_{x}=-\sin \phi \cos \psi-\cos \theta \cos \phi \sin \psi, \\
& l_{y}=\cos \phi \cos \psi-\cos \theta \sin \phi \sin \psi, \\
& l_{z}=\sin \theta \sin \psi .
\end{aligned}
$$

It is straightforward to verify, using standard rotation matrices, that these mutually orthonormal vectors correspond to the orientation and description of the biaxial plate as shown in Fig. 3.

\subsection{A simple shear flow}

As an example, from the early investigations of Carlsson et al. [25] and Leslie [24], consider a biaxial nematic liquid crystal under a simple shear, ignoring for the present 
any boundary influences or elastic contributions. We seek to find all the steady, uniform flow alignment configurations that can occur in simple shear flow; these will necessarily be solutions to the dynamic continuum equations for $\mathbf{n}$ and $\mathbf{m}$ in terms of Euler angles that are constants, which are to be determined. We introduce an induced velocity, relative to a fixed Cartesian coordinate system $O x y z$, of the form

$$
\mathbf{v}=(k z, 0,0),
$$

where $k$ is a non-zero constant, and adopt the forms for $\mathbf{n}$ and $\mathbf{m}$ given in eqs.(3.43) and (3.44). The requirements in eq.(3.36) and the incompressibility condition eq.(3.37) are clearly satisfied. The linear momentum equations in eq.(3.38) reduce, in the presumed absence of external body forces and generalised torques, to a determination of the pressure, which leads to establishing that $p=p_{0}$, where $p_{0}$ is an arbitrary constant that results from the assumed incompressibility. Therefore in order to find complete solutions it only remains to solve the remaining six equations contained in eq.(3.39) and eq.(3.40) for the three Euler angles and three Lagrange multipliers. There are two ways to proceed in general: we can evaluate the Lagrange multipliers by taking the scalar products of these equations with $\mathbf{n}$ and $\mathbf{m}$ and inserting the values for the multipliers back into the equations and then solving them for the orientation angles (a process used below in the next section to identify constant equilibria), or we can eliminate the Lagrange multipliers by taking the scalar product of both sets of equations with $\mathbf{l}$ and then proceed to eliminate $\kappa$ by taking the scalar product of eq.(3.39) with $\mathbf{m}$ and the scalar product of eq.(3.40) with $\mathbf{n}$. Both these techniques, or combinations of them, have different advantages in different circumstances. Here we opt for taking the second approach. Doing so, reveals that

$$
\tilde{\mathbf{g}}^{n} \cdot \mathbf{l}=0, \quad \tilde{\mathbf{g}}^{m} \cdot \mathbf{l}=0, \quad \tilde{\mathbf{g}}^{n} \cdot \mathbf{m}=\tilde{\mathbf{g}}^{m} \cdot \mathbf{n} .
$$

Using the properties eq.(3.36) combined with the expressions for $\tilde{\mathbf{g}}^{n}$ and $\tilde{\mathbf{g}}^{m}$ in equations eq.(3.27) and eq.(3.28) and the result in eq.(3.19), the equations in (3.47) further reduce to $[24]$

$$
\begin{aligned}
\left(\gamma_{1} \mathbf{N}+\gamma_{2} \mathbf{A n}\right) \cdot \mathbf{l} & =0 \\
\left(\lambda_{1} \mathbf{M}+\lambda_{2} \mathbf{A} \mathbf{m}\right) \cdot \mathbf{l} & =0 \\
{\left[\left(\gamma_{1}+\gamma_{3}+\lambda_{1}\right) \mathbf{N}+\left(\gamma_{2}+\gamma_{4}-\lambda_{2}\right) \mathbf{A} \mathbf{n}\right] \cdot \mathbf{m} } & =0 .
\end{aligned}
$$

The general forms for $\mathrm{A}, \mathrm{W}, \mathbf{M}$ and $\mathbf{N}$ based on the directors and the prescribed velocity in eq.(3.46) show that these expressions can be written as, respectively,

$$
\begin{aligned}
\left(1+\tau_{1}\right) n_{x} l_{z} & =\left(1-\tau_{1}\right) n_{z} l_{x}, & & \tau_{1}=\gamma_{2} / \gamma_{1} \\
\left(1+\tau_{2}\right) m_{x} l_{z} & =\left(1-\tau_{2}\right) m_{z} l_{x}, & & \tau_{2}=\lambda_{2} / \lambda_{1} \\
\left(1+\tau_{3}\right) n_{x} m_{z} & =\left(1-\tau_{3}\right) m_{x} n_{z}, & & \tau_{3}=\left(\gamma_{2}+\gamma_{4}-\lambda_{2}\right) /\left(\gamma_{1}+\gamma_{3}+\lambda_{1}\right)
\end{aligned}
$$

under the assumption that $\gamma_{1}, \lambda_{1}$ and $\gamma_{1}+\gamma_{3}+\lambda_{1}$ are non-zero, in accord with the inequalities stated in eq.(3.31).

The expressions for the directors given in eqs.(3.43), (3.44) and (3.45) can now be inserted into these results. After some tedious algebra, three types of solutions can be 
found, which we label as $S_{n}, S_{m}$ and $S_{l}$. They are defined by

$$
\begin{aligned}
& S_{n}:\left\{\begin{array}{l}
\theta=\frac{\pi}{2}, \quad \phi=\frac{\pi}{2}, \quad \tau_{2} \cos (2 \psi)=1, \\
\mathbf{n}=(0,1,0), \quad \mathbf{m}=(-\sin \psi, 0,-\cos \psi), \quad \mathbf{l}=(-\cos \psi, 0, \sin \psi),
\end{array}\right. \\
& S_{m}:\left\{\begin{array}{l}
\phi=0, \quad \psi=\frac{\pi}{2}, \quad \tau_{1} \cos (2 \theta)=1, \\
\mathbf{n}=(\sin \theta, 0, \cos \theta), \quad \mathbf{m}=(0,1,0), \quad \mathbf{l}=(-\cos \theta, 0, \sin \theta),
\end{array}\right. \\
& S_{l}:\left\{\begin{array}{l}
\phi=0, \quad \psi=0, \quad \tau_{3} \cos (2 \theta)=1, \\
\mathbf{n}=(\sin \theta, 0, \cos \theta), \quad \mathbf{m}=(\cos \theta, 0,-\sin \theta), \quad \mathbf{l}=(0,1,0) .
\end{array}\right.
\end{aligned}
$$

It is easy to verify directly that $S_{n}, S_{m}$ and $S_{l}$ satisfy the relations eqs.(3.51) to (3.53), which consequently shows that they are indeed steady state solutions to the dynamic equations in (3.47), as required. These are the solutions that were identified (with variant definitions of the Euler angles) by Carlsson et al. [25] and by Leslie [24]. For these solutions to be possible the magnitudes of the dimensionless parameters $\tau_{1}, \tau_{2}$ and $\tau_{3}$ must all be greater than or equal to unity. When this is the case, as remarked in [24], there are in general four constant values for each of the angles that are determined from the equations in the first lines of the definitions of each solution, although any collection of such four constants really represents two pairs of physically equivalent alignments since values that differ by $\pi$ are physically indistinguishable because the theory is invariant to independent changes of $\operatorname{sign}$ in $\mathbf{n}$ and $\mathbf{m}$. There are therefore six possible steady state alignments in total, two of each type. What is less obvious (full details can be found in [24]) is that these six solutions are the only possible solutions if it is assumed that

$$
\left|\tau_{1}\right|>1, \quad\left|\tau_{2}\right|>1, \quad\left|\tau_{3}\right|>1, \quad \text { and } \quad \tau_{1}\left(1+\tau_{2} \tau_{3}\right) \neq \tau_{2}+\tau_{3}
$$

By means of an illustrative example of six such solutions we can set $\tau_{1}=\tau_{2}=\tau_{3}=2$ so that the inequalities in eq.(3.57) hold and $\theta= \pm \pi / 6$ or $\psi= \pm \pi / 6$ (as appropriate to each case), with the corresponding six solution types labelled $S_{n_{ \pm}}, S_{m_{ \pm}}$and $S_{l_{ \pm}}$, according to the appropriate sign of the orientation angle around the $y$-axis. A straightforward consideration of the Euler angles as defined in Fig. 3 shows that these solutions can be represented schematically as shown in Fig. 4.

Basic stability analyses have been carried out on these six steady state alignments by Leslie [24] and by Carlsson et al. [25]. It was demonstrated in [25] that, for physically feasible parameter values, the three solutions $S_{n_{+}}, S_{m_{+}}$and $S_{l_{+}}$are stable. The solutions $S_{n_{-}}, S_{m_{-}}$and $S_{l-}$ were also shown in [25] to be unstable under the assumptions that

$$
\beta_{2}<0, \quad \alpha_{2}<0, \quad \text { with } \quad\left|\alpha_{2}\right|>\max \left\{\left|\mu_{1}+\mu_{2}\right|,\left|\beta_{2}\right|\right\} .
$$

These results obtained under these conditions, combined with the remarks made by Leslie [24], show that if only one of the parameters $\tau_{1}, \tau_{2}$ and $\tau_{3}$ has magnitude greater than unity, then the corresponding plus sign solution is the only possible stable alignment, provided the remaining two parameters obey a certain additional inequality, as prescribed in eqs.(3.59) to (3.61). In summary, if two of these parameters have magnitude less then unity and the inequalities in eq.(3.58) are satisfied then only one of the 
(a)
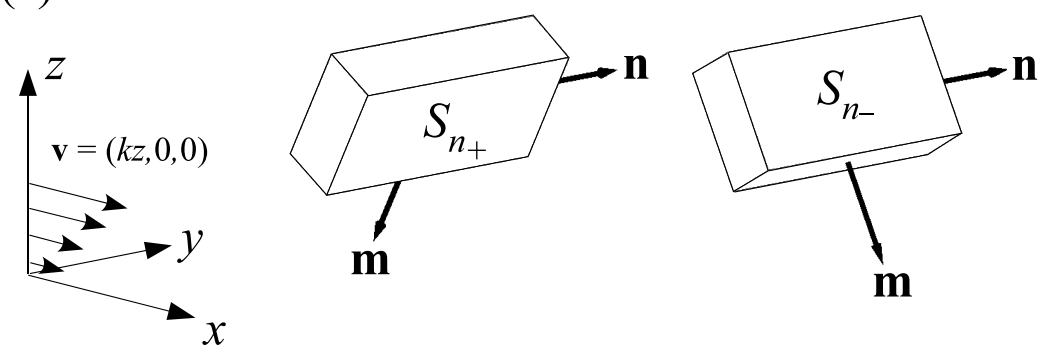

(b)
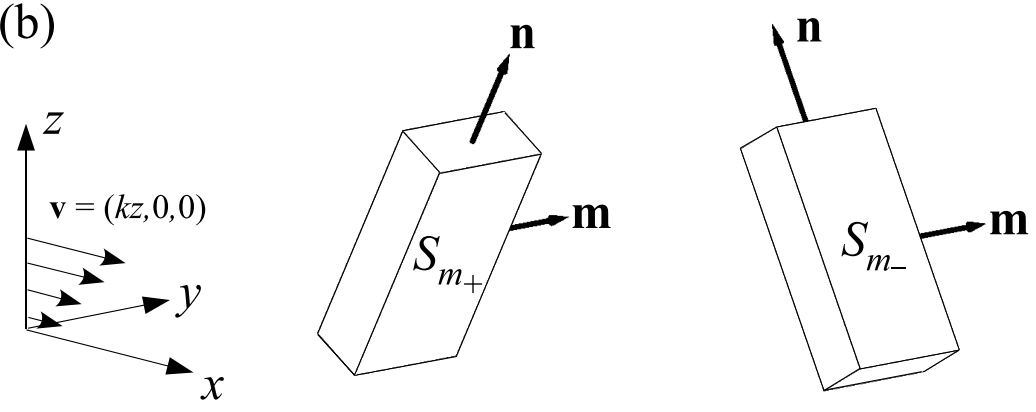

(c)
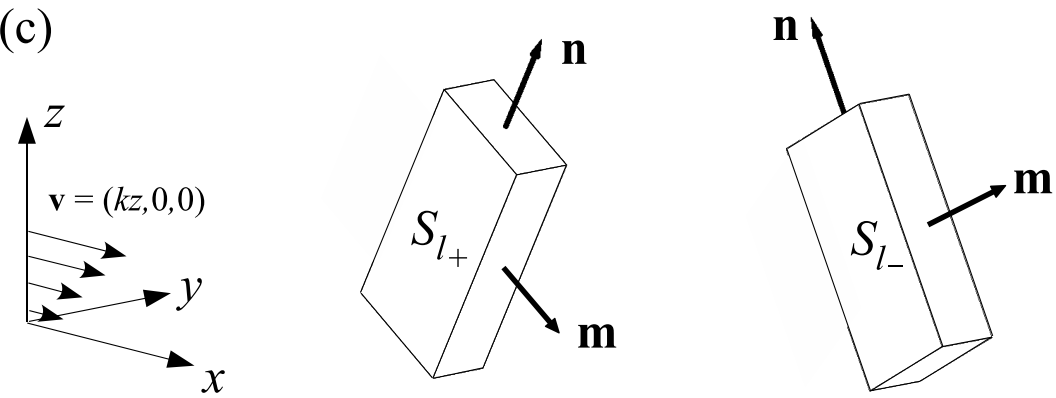

Figure 4: Examples of steady uniform flow alignment under a simple shear with $\mathbf{v}=$ $(k z, 0,0)$. The possible solutions of the forms given in eqs.(3.54) to (3.56) when $\tau_{1}=\tau_{2}=$ $\tau_{3}=2$ are as shown. (a) $S_{n_{ \pm}}: \theta=\frac{\pi}{2}, \phi=\frac{\pi}{2}, \psi= \pm \frac{\pi}{6}$. (b) $S_{m_{ \pm}}: \theta= \pm \frac{\pi}{6}, \phi=0, \psi=\frac{\pi}{2}$. (c) $S_{l_{ \pm}}: \theta= \pm \frac{\pi}{6}, \phi=0, \psi=0$. Under the assumptions stated in eq.(3.58), the alignments with a 'minus' suffix are unstable; those with a 'plus' suffix can be shown to be generally stable under physically feasible parameters, with only one of them being stable if further inequalities on the dimensionless parameters $\tau_{1}, \tau_{2}$ and $\tau_{3}$ are supposed, according to the classifications in eqs.(3.59) to (3.61).

six solutions identified here is stable. In these circumstance, the unique stable alignment can be identified as one of the following three possibilities:

$$
\begin{aligned}
S_{n_{+}}: & \text {stable if }\left|\tau_{1}\right|<1,\left|\tau_{2}\right|>1,\left|\tau_{3}\right|<1 \text { and } \tau_{2}\left(\tau_{1}-\tau_{3}\right)>0, \\
S_{m_{+}}: & \text {stable if }\left|\tau_{1}\right|>1,\left|\tau_{2}\right|<1,\left|\tau_{3}\right|<1 \text { and } \tau_{1}\left(\tau_{2}+\tau_{3}\right)>0, \\
S_{l_{+}}: & \text {stable if }\left|\tau_{1}\right|<1,\left|\tau_{2}\right|<1,\left|\tau_{3}\right|>1 \text { and } \tau_{3}\left(\tau_{1}-\tau_{2}\right)>0 .
\end{aligned}
$$

Other possible conditions for stability have been discussed by Leslie [24] and by Carlsson et al. [25]. Of course, if all of $\tau_{1}, \tau_{2}$ and $\tau_{3}$ have magnitude less than unity then no 
steady flow alignment is possible.

\section{Equilibrium equations}

The equations summarised in Section 3.3 are for the dynamics of incompressible biaxial liquid crystals. In the following subsections we summarise the equilibrium equations and give an application which examines possible sample alignments that can be induced by an external magnetic field.

\subsection{The equilibrium equations}

The equilibrium equations for incompressible biaxial nematics can be extracted from the dynamic equations. In this case there is no flow and all viscous contributions can be neglected; the balance of linear momentum in eq.(3.38) then simply yields an identification of the pressure $p$, which can be calculated if required but otherwise may be neglected, unless there is a desire to compute forces via eq.(3.38). This is because the linear momentum equations, in view of eq.(2.19), reduce to

$$
\left(p+w_{e l}-\Psi\right)_{, i}=0
$$

where $\Psi$ is any or all of the potentials introduced in Section 2.2, which implies that

$$
p+w_{e l}-\Psi=p_{0},
$$

where $p_{0}$ is an arbitrary constant. The remaining equilibrium equations reduce, in conjunction with eq.(3.36), to the corresponding coupled Euler-Lagrange equations for the minimisation of the appropriate energy, obtained by omitting the terms $\tilde{\mathbf{g}}^{n}$ and $\tilde{\mathbf{g}}^{m}$ in eqs.(3.39) and (3.40). Such modified versions of eqs.(3.36), (3.39) and (3.40) give rise to nine equations for the remaining nine unknowns: six unknowns from the components of $\mathbf{n}$ and $\mathbf{m}$ and three from the Lagrange multipliers $\gamma, \tau$ and $\kappa$. For the sake of completeness, we state the full set of these equilibrium equations here for convenience. They consist of the constraints on $\mathbf{n}$ and $\mathbf{m}$

$$
n_{i} n_{i}=1, \quad m_{i} m_{i}=1, \quad n_{i} m_{i}=0,
$$

and the coupled system of equations

$$
\begin{aligned}
\left(\frac{\partial w_{e l}}{\partial n_{i, j}}\right)_{, j}-\frac{\partial w_{e l}}{\partial n_{i}}+G_{i}^{n} & =\gamma n_{i}+\kappa m_{i} \\
\left(\frac{\partial w_{e l}}{\partial m_{i, j}}\right)_{, j}-\frac{\partial w_{e l}}{\partial m_{i}}+G_{i}^{m} & =\tau m_{i}+\kappa n_{i}
\end{aligned}
$$

where $\mathbf{G}^{n}$ and $\mathbf{G}^{m}$ are the generalised torques discussed in Section 2.2.

The equilibrium equations for uniaxial nematics may be obtained by further setting $\tau=\kappa=0$ and neglecting all the terms that involve $\mathbf{m}$. 


\subsection{Alignment induced by a magnetic field}

We give an elementary application of the equilibrium equations so that the procedure to evaluate the Lagrange multipliers is demonstrated. This methodology also ensures that all possible solutions are identified so that the paths to further analysis can be made clear (see also Chapter 8 by Photinos).

Consider the example given by Carlsson and Leslie [27] when a magnetic field $\mathbf{H}=$ $H \mathbf{e}_{z}$, with $H=|\mathbf{H}|$, is applied across a sample of biaxial nematic liquid crystal where elastic effects and the influence of boundaries may be neglected, as pictured in Fig. 5 . The magnetic energy density is given by eq.(2.13), which for this case with the field in

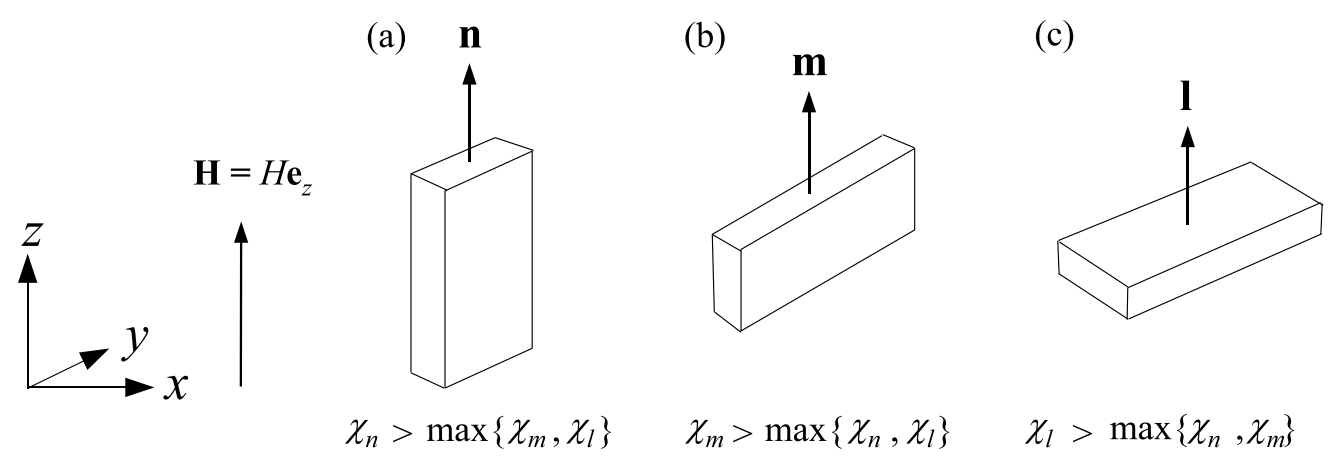

Figure 5: A magnetic field $\mathbf{H}=H \mathbf{e}_{z}$ is applied across a biaxial nematic as shown. (a) When $\chi_{n}>\max \left\{\chi_{m}, \chi_{l}\right\}$ then the major director $\mathbf{n}$ prefers to align with the field. (b) If $\chi_{m}>\max \left\{\chi_{n}, \chi_{l}\right\}$ then the minor director $\mathbf{m}$ aligns parallel to the field. (c) Similarly, $\mathbf{l}$ aligns with the field if $\chi_{l}>\max \left\{\chi_{m}, \chi_{n}\right\}$. In all three cases the two directors orthogonal to the field have indeterminate, but mutually orthogonal, directions within a plane perpendicular to $\mathbf{H}$.

the $z$-direction is given explicitly by

$$
w_{m}=-\frac{1}{2} \mu_{0}\left[\chi_{n l}\left(H n_{z}\right)^{2}+\chi_{m l}\left(H m_{z}\right)^{2}+\chi_{l} H^{2}\right],
$$

where, in an obvious notation, only the z-components of the major and minor directors can enter directly into the energy. The generalised torques in this example are given by equation eq.(2.17). Inserting these into the equilibrium equations (4.4) and (4.5), and neglecting the elastic energy $w_{e l}$, gives

$$
\begin{aligned}
\mu_{0} \chi_{n l}(\mathbf{n} \cdot \mathbf{H}) H_{i} & =\gamma n_{i}+\kappa m_{i}, \\
\mu_{0} \chi_{m l}(\mathbf{m} \cdot \mathbf{H}) H_{i} & =\tau m_{i}+\kappa n_{i} .
\end{aligned}
$$

Taking the scalar products of these equations with $\mathbf{n}$ and $\mathbf{m}$, and using the orthonormal properties in eq.(4.3), reveals that the Lagrange multipliers must satisfy the relations

$$
\begin{aligned}
\mu_{0} \chi_{n l}(\mathbf{n} \cdot \mathbf{H})^{2} & =\gamma, \\
\mu_{0} \chi_{m l}(\mathbf{m} \cdot \mathbf{H})^{2} & =\tau, \\
\mu_{0} \chi_{n l}(\mathbf{n} \cdot \mathbf{H})(\mathbf{m} \cdot \mathbf{H}) & =\kappa=\mu_{0} \chi_{m l}(\mathbf{n} \cdot \mathbf{H})(\mathbf{m} \cdot \mathbf{H}) .
\end{aligned}
$$


For the present discussion it can be assumed that $\chi_{n l} \neq \chi_{m l}$, which is generally going to be the case except possibly in certain disk-like biaxial nematics, as mentioned briefly in Section 3.2. From eq.(4.11) it is seen that for consistency we must have (i) $\mathbf{m} \cdot \mathbf{H}=0$ and $\mathbf{n} \cdot \mathbf{H} \neq 0$, or, (ii) $\mathbf{n} \cdot \mathbf{H}=0$ and $\mathbf{m} \cdot \mathbf{H} \neq 0$, or (iii) $\mathbf{n} \cdot \mathbf{H}=\mathbf{m} \cdot \mathbf{H}=0$. We examine each of these in turn.

Case (i): $\mathbf{m} \cdot \mathbf{H}=0$ and $\mathbf{n} \cdot \mathbf{H} \neq 0$. In this case $\mathbf{m}$ is perpendicular to $\mathbf{H}$ with $\gamma$ given by eq.(4.9) and $\tau=\kappa=0$. Eq.(4.8) is then automatically satisfied and a brief inspection of eq.(4.7) reveals the only solution for the major director, namely, $\mathbf{n}=(0,0,1)$. Thus both $\mathbf{m}$ and $\mathbf{l}$ must be perpendicular to $\mathbf{H}$ and $\mathbf{n}$. The third components of $\mathbf{m}$ and $\mathbf{l}$ must therefore satisfy $m_{z}=l_{z}=0$ and therefore the energy density eq.(4.6) reduces to, using the definition eq.(2.6)

$$
w_{m}=-\frac{1}{2} \mu_{0} \chi_{n} H^{2} .
$$

Case (ii): $\mathbf{n} \cdot \mathbf{H}=0$ and $\mathbf{m} \cdot \mathbf{H} \neq 0$. This is similar to the first case. Here $\mathbf{n}$ is perpendicular to $\mathbf{H}$ with $\tau$ given by eq.(4.10) and $\gamma=\kappa=0$. Eq.(4.7) is then automatically satisfied and eq.(4.8) reveals the only solution for the minor director to be $\mathbf{m}=(0,0,1)$. Thus, by analogy with the previous argument, the third components of $\mathbf{n}$ and $\mathbf{l}$ must be zero. The energy density eq.(4.6) is then

$$
w_{m}=-\frac{1}{2} \mu_{0} \chi_{m} H^{2} .
$$

Case (iii): $\mathbf{n} \cdot \mathbf{H}=\mathbf{m} \cdot \mathbf{H}=0$. Here, $\gamma=\tau=\kappa=0$ and eqs.(4.7) and (4.8) are automatically satisfied. Both $\mathbf{n}$ and $\mathbf{m}$ are perpendicular to the magnetic field and so $\mathbf{l}$ must be parallel to $\mathbf{H}$, giving $\mathbf{l}=(0,0,1)$. The energy density in this case is

$$
w_{m}=-\frac{1}{2} \mu_{0} \chi_{l} H^{2} .
$$

These results now lead to three possibilities. If $\chi_{n}>\max \left\{\chi_{m}, \chi_{l}\right\}$ then the least energy is given by eq.(4.12) and the major director $\mathbf{n}$ must align with the field, as shown in Fig. 5(a). Similarly, if $\chi_{m}>\max \left\{\chi_{n}, \chi_{l}\right\}$ then the least energy is given by eq.(4.13) and the minor director $\mathbf{m}$ aligns parallel to the field, as shown in Fig. 5(b). Lastly, if $\chi_{l}>\max \left\{\chi_{n}, \chi_{m}\right\}$ then the minimum energy is given by eq.(4.14) and $\mathbf{l}$ is parallel to the field, as in Fig. 5(c). In all three possibilities the precise alignment of the two directors that are perpendicular to the field is indeterminate, except that they are mutually perpendicular and lie in a plane perpendicular to $\mathbf{H}$. It is this indeterminacy that evidently led Chandrasekhar [41] to suggest that biaxial nematic systems may be aligned by the use of two crossed electric and magnetic fields, as subsequently investigated by Carlsson and Leslie [27], who managed to identify stable orientations of the directors that depend upon the relative magnitudes of the magnetic and dielectric anisotropies. These authors also identified bistability in special cases. The interested reader is referred to the exhaustive details of these results which have been tabulated in [27]. A further more detailed analysis of crossed fields and the possible biaxial alignments that are available can be found in the work of Carlsson et al. $[25,26]$.

\section{Conclusion}

This article has summarised the key features of the continuum theory for biaxial nematic liquid crystals. The general model variables and the associated elastic, electric and 
magnetic energies for biaxial nematics have been described in Section 2 and the dynamic theory and continuum equations were reviewed in Section 3, with a brief introduction to the equilibrium theory in Section 4.

The dynamic theory requires twelve independent viscosity coefficients; nevertheless, despite this complexity, three main rotational viscosities were identified and interpreted schematically in Fig. 2. An example of a simple shear flow, in Section 3.5, demonstrated some of the basic techniques used for applying this theory to problems in dynamics. A description using standard Euler angles, shown in Fig. 3, proved particularly convenient. Possible steady uniform alignments were found for this simple shear, displayed in Fig. 4, and various mechanisms for identifying the physically relevant stable solutions were discussed in terms of the relative magnitudes of various combinations of viscosities. These ideas can, of course, be extended to much more complex geometries and flow alignment problems.

In Section 4 an application of the equilibrium equations was given to model possible static alignments under the influence of a magnetic field. The results developed in that section can be transformed to analogous results for an electric field by replacing $w_{m}$ with the electric energy density $w_{e}$, given by eq. (2.21), and interchanging the notation in an obvious manner. Three possible alignments were identified that depend on the relative magnitudes of the magnetic susceptibilities in the magnetic field case. In each alignment, the biaxial plate will have one of its directors $\mathbf{n}, \mathbf{m}$ or $\mathbf{l}$ align parallel to the field. No matter which director aligns with the field, there is always an indeterminacy in the orientation within a plane of the remaining two directors, as shown in Fig. 5. This indeterminacy may be resolved by considering crossed electric and magnetic fields, as mentioned in Section 4.

\section{References}

[1] M. J. Freiser. Ordered states of a nematic liquid. Phys. Rev. Lett., 24:1041-1043, 1970.

[2] M. J. Freiser. Successive transitions in a nematic liquid. Mol. Cryst. Liq. Cryst., 14:165-182, 1971.

[3] L. J. Yu and A. Saupe. Observation of a biaxial nematic phase in potassium laurate-1-decanol-water mixtures. Phys. Rev. Lett., 45(12):1000-1003, 1980.

[4] J. Malthête, L. Liebert, A. M. Levelut, and Y. Galerne. Nématic biaxe thermotrope. C. R. Acad. Sci. Paris, 303:1073-1076, 1986.

[5] S. Chandrasekhar, B. K. Sadashiva, B. R. Ratna, and V. N. Raja. A biaxial nematic liquid crystal. Pramana, 3:L491-L494, 1988.

[6] S. Chandrasekhar, B. R. Ratna, B. K. Sadashiva, and V. N. Raja. A thermotropic biaxial nematic liquid crystal. Mol. Cryst. Liq. Cryst., 165:123-130, 1988.

[7] K. Praefcke, B. Kohne, B. Gündogan, D. Singer, D. Demus, S. Diele, G. Pelzl, and U. Bakowsky. News on nematic-biaxial liquid crystals. Mol. Cryst. Liq. Cryst., 198:393-405, 1991. 
[8] G. R. Luckhurst. Biaxial nematic liquid crystals: fact or fiction? Thin Solid Films, 393:40-52, 2001.

[9] A. Saupe. Elastic and flow properties of biaxial nematics. J. Chem. Phys., 75:51185124, 1981.

[10] M. Liu. Hydrodynamic theory of biaxial nematics. Phys. Rev. A, 24:2720-2726, 1981.

[11] U. D. Kini. Isothermal hydrodynamics of orthorhombic nematics. Mol. Cryst. Liq. Cryst., 108:71-91, 1984.

[12] E. Govers and G. Vertogen. Elastic continuum theory of biaxial nematics. Phys. Rev. A, 30(4):1998-2000, 1984.

[13] E. Govers and G. Vertogen. Erratum: Elastic continuum theory of biaxial nematics [Phys. Rev. A 30, 1998 (1984)]. Phys. Rev. A, 31(3):1957, 1985.

[14] E. Govers and G. Vertogen. Fluid dynamics of biaxial nematics. Physica A, 133:337$344,1985$.

[15] A. Chauré. Théorie hydrodynamique des cristaux liquides nématiques biaxes. Int. J. Eng. Sci., 23(8):797-807, 1985.

[16] F. M. Leslie, J. S. Laverty, and T. Carlsson. Continuum theory for biaxial nematic liquid crystals. Q. Jl. Mech. Appl. Math., 45:595-606, 1992.

[17] F. M. Leslie and T. Carlsson. Flow alignment in biaxial and discotic nematics. Mol. Cryst. Liq. Cryst., 292:113-122, 1997.

[18] L. A. Madsen, T. J. Dingemans, M. Nakata, and E. T. Samulski. Thermotropic biaxial nematic liquid crystals. Phys. Rev. Lett., 92:145505, 2004.

[19] B. R. Acharya, A. Primak, and S. Kumar. Biaxial nematic phase in bent-core thermotropic mesogens. Phys. Rev. Lett., 92(14):145506, 2004.

[20] K. Severing and K. Saalwächter. Biaxial nematic phase in a thermotropic liquidcrystalline side-chain polymer. Phys. Rev. Lett., 92:125501, 2004.

[21] K. Merkel, A. Kocot, J. K. Vij, R. Korlacki, G. H. Mehl, and T. Meyer. Thermotropic biaxial nematic phase in liquid crystalline organo-siloxane tetrapodes. Phys. Rev. Lett., 93:237801, 2004.

[22] J. You, J. Y. Yung, K. Rhie, V. M. Pergamenshchik, and S. T. Shin. Macroscopic properties of the nematic phase of boomerang-shaped "C7": Evidence of biaxiality. J. Korean Phys. Soc., 52:342-349, 2008.

[23] G. R. Luckhurst. A missing phase found at last? Nature, 430(6998):413-414, 2004.

[24] F. M. Leslie. Flow alignment in biaxial nematic liquid crystals. J. Non-Newtonian Fluid Mech., 54:241-250, 1994. 
[25] T. Carlsson, F. M. Leslie, and J. S. Laverty. Flow properties of biaxial nematic liquid crystals. Mol. Cryst. Liq. Cryst., 210:95-127, 1992.

[26] T. Carlsson, F. M. Leslie, and J. S. Laverty. Biaxial nematic liquid crystals - flow properties and evidence of bistability in the presence of electric and magnetic fields. Mol. Cryst. Liq. Cryst., 212:189-196, 1992.

[27] T. Carlsson and F. M. Leslie. Behaviour of biaxial nematics in the presence of electric and magnetic fields: Evidence of bistability. Liq. Cryst., 10:325-340, 1991.

[28] P. K. Karahaliou, A. G. Vanakaras, and D. J. Photinos. Symmetries and alignment of biaxial nematic liquid crystals. J. Chem. Phys., 131:124516, 2009.

[29] P. G. de Gennes and J. Prost. The Physics of Liquid Crystals. Oxford University Press, Oxford, second edition, 1993.

[30] I. W. Stewart. The Static and Dynamic Continuum Theory of Liquid Crystals. Taylor and Francis, London and New York, 2004.

[31] W. H. de Jeu. Physical Properties of Liquid Crystalline Materials. Gordon and Breach, New York, 1990.

[32] J. L. Ericksen. Equilibrium theory of liquid crystals. Adv. Liq. Cryst., 2:233-298, 1976.

[33] F. M. Leslie. Continuum theory for nematic liquid crystals. Continuum Mech. Thermodyn., 4:167-175, 1992.

[34] L. E. Malvern. Introduction to the Mechanics of a Continuous Medium. PrenticeHall, Englewood Cliffs, New Jersey, 1969.

[35] R. J. Atkin and N. Fox. On the frame-dependence of stress and heat flux in polar fluids. Z. Angew. Math. Phys., 24:853-860, 1973.

[36] F. M. Leslie. Continuum theory for liquid crystals. In D. Demus, J. Goodby, G. W. Gray, H. W. Spiess, and V. Vill, editors, Handbook of Liquid Crystals, volume 1, pages 25-39. Wiley-VCH, Weinheim, Germany, 1998.

[37] F. M. Leslie. Some constitutive equations for anisotropic fluids. Q. Jl. Mech. Appl. Math., 19:357-370, 1966.

[38] F. M. Leslie. Some constitutive equations for liquid crystals. Arch. Rat. Mech. Anal., 28:265-283, 1968.

[39] D. A. Dunmur, A. Fukuda, and G. R. Luckhurst, editors. Physical Properties of Liquid Crystals: Nematics. EMIS Datareviews Series No. 25. The Institution of Electrical Engineers (INSPEC), London, 2001.

[40] J. L. Synge and B. A. Griffith. Principles of Mechanics. McGraw-Hill Kogakusha, New York, third edition, 1959.

[41] S. Chandrasekhar. 8th Liquid Crystal Conference of Socialist Countries, Krakow, Poland. (Unpublished oral communication), 1989. 